\title{
THE MEASUREMENT OF ELECTRICAL CONDUCTIVITY IN
}

\section{METALS AND ALLOYS AT HIGH TEMPERATURES.}

\author{
By John L. Haughton, D.Sc.,
}

From the National Physical Laboratory.

\section{A Paper read before The Faraday Society, June 14, 1920, Professor} Alfred W. PORTER, D.Sc., F.R.S., Vice-President, in the Chair.)

The measurement of electrical conductivity of metals at ordinary temperatures has been carried to such a degree of perfection that it. is comparable in accuracy with the measurement of mass or of length. Such determinations have been carried out, probably with but small loss of accuracy, at temperatures of the order of $100^{\circ} \mathrm{C}$. by immersing the specimen in an oil bath. The measurement of conductivity at much higher temperatures-up to $1,000^{\circ} \mathrm{C}$.- -has, however, not received nearly as much attention, and the purpose of this paper is to describe an apparatus with which such determinations can readily be carried out.

The apparatus was designed primarily to determine the electrical conductivity of a series of alloys at varying temperatures with a view to obtaining some information on their constitution. The whole theory of the study of electrical conductivity of alloys as an aid to the establishment of their equilibrium diagrams has been very fully discussed by Guertler, ${ }^{*}$ and it is only necessary to state that this: method, as well as the study of certain other physical properties, has the great advantage that measurements can be carried out as slowly as may be necessary to attain complete equilibrium. This is manifestly impossible in the case of thermal analysis, as there is a minimum speed at which thermal curves can be taken, a speed determined partly by the inaccuracy introduced in timing a very slowly moving galvanometer spot, and partly by the exceedingly small effect produced by a small heat evolution spread over a long time: This applies to any set of observations relating to a rate of change, as is the case in thermal curves, and not a steady condition, as in the case of electrical conductivity. In addition to this, the use of thermal curves is limited to the determination of phase boundaries which do not depart very markedly from the horizontal-limits of solubility, for example, can rarely be detected on thermal curves-while this is not the case with electrical conductivity measurements.

It must not be inferred from these remarks that the author ccnsiders that the electrical conductivity method is likely to supersede the thermal curve method as an implement for determining the constitution of alloys. On the contrary, he is convinced that the

* W. Guertler. The Electrical Conductivity and Constitution of Alloys. Int. Inst. Met., Vol. 6 (1011), p. 135. 
most important place will always be shared by the method of thermal snalysis and the examination of annealed and quenched specimens by the microscope. The majority of reactions occur sufficiently readily for their temperature to be determined by a comparison of heating and cooling curves. Thermal curves can be taken so quickly, easily and accurately, provided suitable methods are employed with reasonable care, that it is probable that the thermal method will always be of prime importance in the investigation of alloy systems.

The determination of the specific conductivity of a series of alloys at a certain temperature, while giving very valuable results, has but a limited application, for it is necessary to know the dimensions of the specimen accurately in order to determine the specific conductivity. To secure this, it is necessary not only to obtain the specimen in a form suitable for accurate measurement, but also to secure the absence of blowholes, and in practice this limits the application of the method to those alloys which can be drawn or rolled, or at least machined. But, instead of measuring the specific condlictivity, it is possible to measure the temperature coefficient of electrical resistance of the alloys, and this can be done without any knıwledge of the dimensions of the sample. The curve connecting temperature coefficient and composition is then plotted, and breaks in this curve indicate the composition at which phase changes occur in the alloys. Another method is to observe the conductivity at a number of temperatures, and to plot a curve connecting conductivity and temperature. Changes in the direction of this curve show the temperatures of phase changes in the alloy, and if a series of such curves be taken with different alloys, the points thus obtained can be plotted on the equilibrium diagram in the same way as points obtained from thermal curves.

The methods of measuring electrical conductivity at ordinary temperatures need not be dealt with here, as reference can be made to any text book on electricity for a description of the methods employed. For use in constitutional work, the problem is simplified by the fact that absolute values are, generally, not required, but is much complicated by the necessity of working at high temperatures. The difficulties introduced by this arise from the necessity of obtain. ing uniformity of temperature in the specimen, and from the fact that there is an unavoidable temperature gradient in the leads to the specimen.

The apparatus described below was designed by the author, and has been in use in the Metallurgy Department of the National Phy. sical Laboratory during the past year with considerable success.

The principle on which the apparatus works is the measurement of the voltage drop along a fixed length of the specimen, through which a constant current is passing. This voltage drop is propo:tional to the resistance between the points of contact so long as the ourrent is kept constant. In order to ensure this, the same current is passed through a constantan strip, whose resistance can be arranged to be of the same order as that of the specimen, and is accurately known, and the voltage drop along this resistance is measured with the same instrument and almost at the same time as that along the specimen. In this way, not only is the constancy of current checked (this being adjusted by means of a rheostat when necessary), but 


\section{MEASUREMENT OF ELECTRICAL CONDUCTIVITY}

also the instrument which is reading the voltage drop is calibrated directly in some multiple of ohms. The details of the arrangement are as follows. The specimen (which is in a specially designed furnace) is in series with a standard resistance and a 2-volt accumulator. By means of a potentiometer or a galvanometer, the voltage drop across the specimen is compared with that across the standard resistance. The connections are shown diagrammatically in Fig. I, where $R x$ represents the specimen whose conductivity is to be measured. $B$ is a 2-volt accumulator sending a current through an ammeter $M$, a series of resistances $R f$ and a low resistance slide wire $R_{r}$ for regulating the current, a standard resistance $R s$, a switch $S_{\text {, }}$

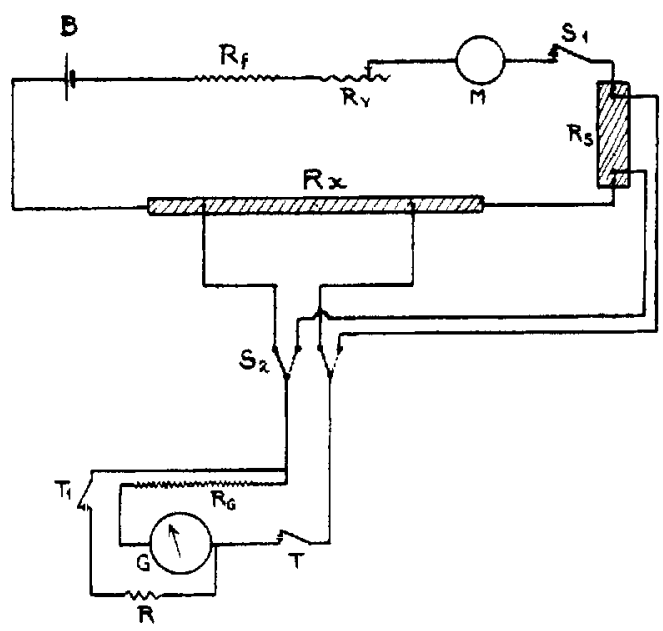

FIG. I.

and the specimen $\mathrm{Rx}$. From two fixed points on Rs, whose distance apart is such that the resistance between them is $0.002 \mathrm{ohms}$, wires are led to a two-way, double-pole switch $\mathbf{S}_{2}{ }^{*}$ Two leads from fixed points in the specimen $R x$ are also connected to this switch, by means of which either these leads or the wires from $R$ s can be connected to a potentiometer or galvanometer and the voltage drop across $\mathrm{Rs}$ compared with that across $\mathrm{Rx}$. While the use of a potentiometer is probably to be recommended where absolute values are required, or where a very high accuracy is sought (particularly if the resistance of the specimen and the standard are very different), the galvanometer is much easier to operate, especially when the resistance of the specimen is much affected by small changes of temperature. Obtaining a balance on the potentiometer takes very much longer than reading a galvanometer deflection. This is of some importance, as the accuracy of the method depends, amongst other things, upon the assumption that the current from the accumulator

"Other tappings, giving different resistances, are also taken from Rs, but, for the sake of simplifying the description, only this one is referred to here. The resistance used should be of the same order as that of the specimen. 
does not change between the readings of $R s$ and $R x$ : the time between these readings should therefore be kept as short as possible. The galvanometer circuit contains a tumbler switch $T$ and a resistance $\mathrm{Rg}$, of about $1,500 \mathrm{ohms}$; the galvanometer can be shunted through a further small resistance $R$ by means of the switch $T_{1}$.

Fig. 2 is a wiring diagram of the apparatus, the wires below the board on which the apparatus is mounted being shown in dotted lines.

It is of the utmost importance that the potential leads from Rs and Rx should be thoroughly insulated from all other leads, as any leakage would be fatal to accuracy. To ensure this, all the leads on the apparatus consist of very heavily insulated copper wire, and even the insulation of the potential leads is not allowed to come into contact with the other wires or with the board on which the apparatus is mounted. All terminals are carried on ebonite blocks, those connected to the potential leads being on separate blocks from the others The aceumulator stands in a trough of paraffin wax.

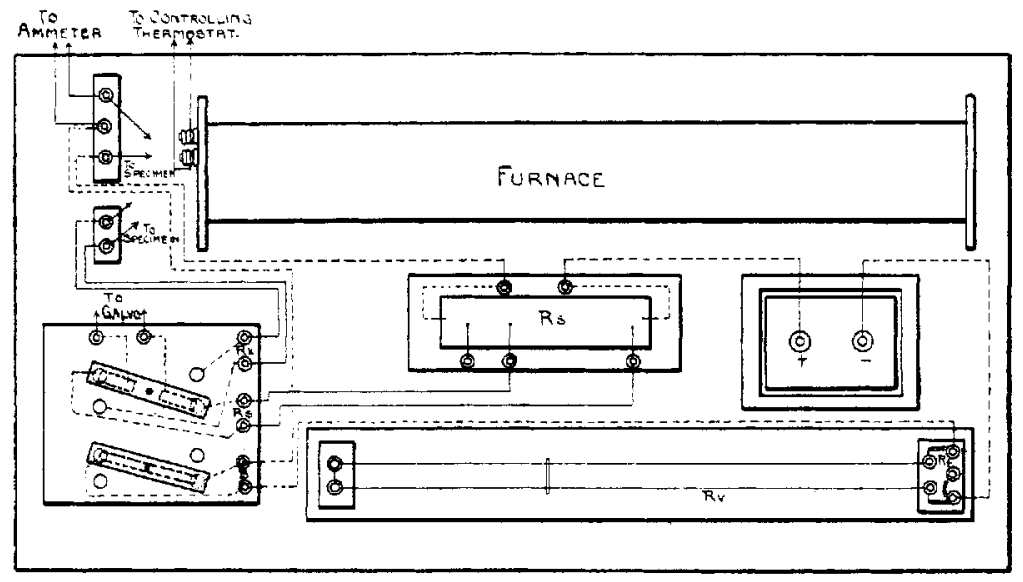

FIG. 2 .

The apparatus is used as follows:-When the specimen, which is in the special furnace described below, is at the desired temperature, the two-way switch $S_{2}$ is set so that it connects the standard resistance, $R s$, with the galvanometer circuit, whose switch, $T$, is then closed. The resistances $\mathrm{Rf}$ and $\mathrm{Rv}$ are then adjusted until such a current is flowing that the drop of potential across the 0.002 ohms of Rs causes a deflection of the galvanometer spot of $200 \mathrm{divi}$ sions. When this is so, one scale division is equal to $10{ }^{5} \mathrm{ohms}$. The switch $T$ is then opened, $S_{2}$ switched over to the leads from the specimen, and $T_{1}$ and $T^{\prime}$ closed. The object of $T_{1}$ is to protect the galvanometer from injury in the event of the specimen being broken, in which case the full voltage from the battery would be applied to the galvanometer terminals. If the specimen be intact, the deflection indicated by the galvanometer is very small, and $T_{1}$ may be opened. The galvanometer deflection will then give the resistance of the specimen in hundredths of a milli-ohm \pm the sum of the various 
currents due to thermal electromative forces in the circuit. In order to find what is the effect of these, the switch $S_{1}$ is now opened, and any deflection of the galvanometer will be due to the combined or opposed effects of the thermal E.M.F.s. The value of this deflection is applied as a correction (either additive or subtractive, as the case may be), to the value obtained for the resistance of the specimen. When the rate of heating or cooling is slow, this correction is rarely greater than one or two scale divisions with the furnace described below, but if the rate of change of temperature be very great, a much larger correction may be found, probably due to a temperature gradient in the specimen. The presence of any thermal E.M.F. in the circuit from the standard resistance is observed by switching $\mathbf{S}_{2}$ on the standard resistance and noting the deflection obtained on the galvanometer scale while $\mathbf{S}_{1}$ is open. Generally speaking, this does not exceed half a division.

The temperature of the specimen is measured by means of a thermo-couple placed at the middle of the specimen and connected to a potentiometer. The scale of the galvanometer used with this potentiometer is placed above the one belonging to the conductivity galvanometer, and the readings are taken in the following order:-

(1) The temperature.

(2) The defection due to the potential drop in Rs.

(3) The deflection due to the potential drop in $\mathrm{Rx}$.

(4) The thermal E.M.F. in Rx.

(5) The temperature.

Readings (1) and (5) should be the same.

If the deflection due to the potential drop in Rs has altered by a very small amount from the original setting, a correction can be applied to the reading of $\mathrm{Rx}$, but it is best to keep the reading of $R$ s as near its correct value as possible.

It may be well at this point to consider the comparative merits of this method of measuring resistance with other possible ones.

(1) The resistance of a circuit can be determined by measuring the current produced in the circuit on the application of a definite E.M.F. to the ends of the circuit. Since, however, the measuring instruments must be outside the furnace, the circuit must include leads to the instruments, and these would have a temperature gradient in them. This would render it extremely difficult to calculate the correction which would be required for the resistance of the leads. Further, the resistance of the specimen would, in general, have to be small compared with the resistance of the leads, as it is necessary to use a specimen which is very short compared with the length of the furnace if a high degree of temperature uniformity is required in the former. Thus we have to measure the change in a small resistance by measuring the change in the sum of this small resistance + a much larger one, which itself is changing. This is by no means an ideal condition. It would be possible to have a dummy lead passing through the furnace close to the other leads and as similar to them in every respect as possible, and by measuring the resistance of these dummy leads almost at the same time as the resistance of the real leads and the specimen to deduce the resistance of the specimen, but this introduces possibilities of serious errors. Slight resistances at contacts anywhere in the circuit will also introduce 
very large errors, as in this method the total resistance of the circuit must be kept low. In the potentialdrop method, on the other hand, the total resistance in the galvanometer circuit may be many hundreds of ohms, and therefore the introduction of a small resistance in the lead is negligible.

(2) A much better way of measuring the resistance of a circuit than the voltmeter-ammeter method is the use of a bridgepreferably, in the case where the resistance is small, a Kelvin double bridge. This method has one advantage over that described in this paper, viz., that the constancy of the current flowing through the circuit is unimportant. Indeed, where accưrate absolute values are required, it is probable that it would be the right method to use. On the other hand, to get a hgh sensitivity, it s necessary to pass a comparatvely heavy current through the specimen. This will introduce considerable difficulties, and probable errors, in the measurement of the temperature of the specimen. The use of a heavy current will also increase the probable errors due to Peltier and Thomson effects.

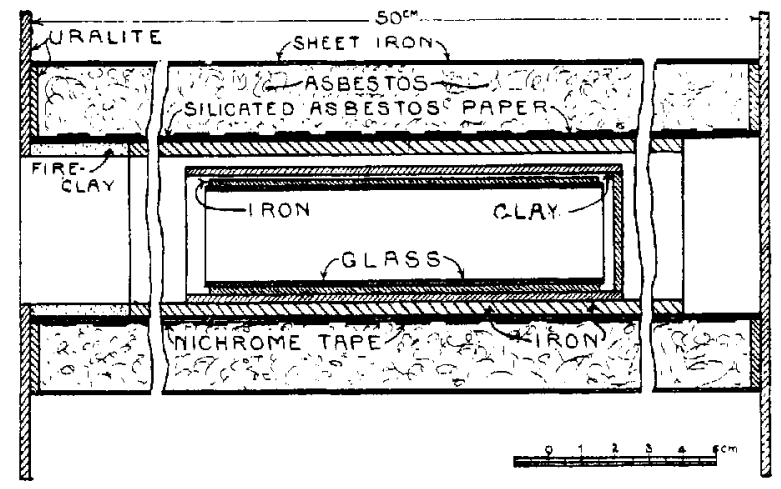

FIG. 3 .

It is of the utmost importance that the temperature of the specimen should be as uniform as possible along its whole length, and to ensure this the furnace described below was designed. The use of iron in its construction limits its use to temperatures not exceeding about $700^{\circ} \mathrm{C}$., but for higher temperatures this can be replaced by an alloy, such as " nichrome," designed to stand high temperatures.

The furnace consists of an iron tube $5 \mathrm{~cm}$. in diameter and $45 \mathrm{~cm}$ long, over which are wrapped several layers of asbestos paper soaked in sodium silicate. (Fig. 3.) This wrapping projects well beyond the end of the iron tube, and, when the sodium silicate is dry, has sufficient rigidity to carry a winding of nichrome tape, even where it is not supported by the iron. The whole furnace is enclosed in a casing of iron $10 \mathrm{~cm}$. in diameter, the space between this and the asbestos tube being filled with magnesia lagging. By thus carrying the winding well beyond the iron tube, the end effect is considerably reduced, and this, together with the comparatively good thermal conductivity of the iron, gives to the furnace a reasonable 
degree of uniformity of temperature. Indeed, the temperature wass found to be constant to $1^{\circ} \mathrm{C}$. over a length of $11 \mathrm{cms}$., the measurements being made by means of a base-metal thermo-couple in the empty furnace.

It has been shown by Kelvin and others that the best way to obtain a uniform temperature in an enclosed space is to have a series of alternate conductors and insulators between the source of heat and the space-a principle which has been partially carried out in this furnace. Inside the iron tube, which is a heat conductor, surroundr $\mathrm{l}$ by the insulating asbestos, is a clay tube $13 \mathrm{cms}$. long, oneend of which is permanently closed. Within this is an iron tube, a. little shorter than the clay one, into which fits another clay or glass.

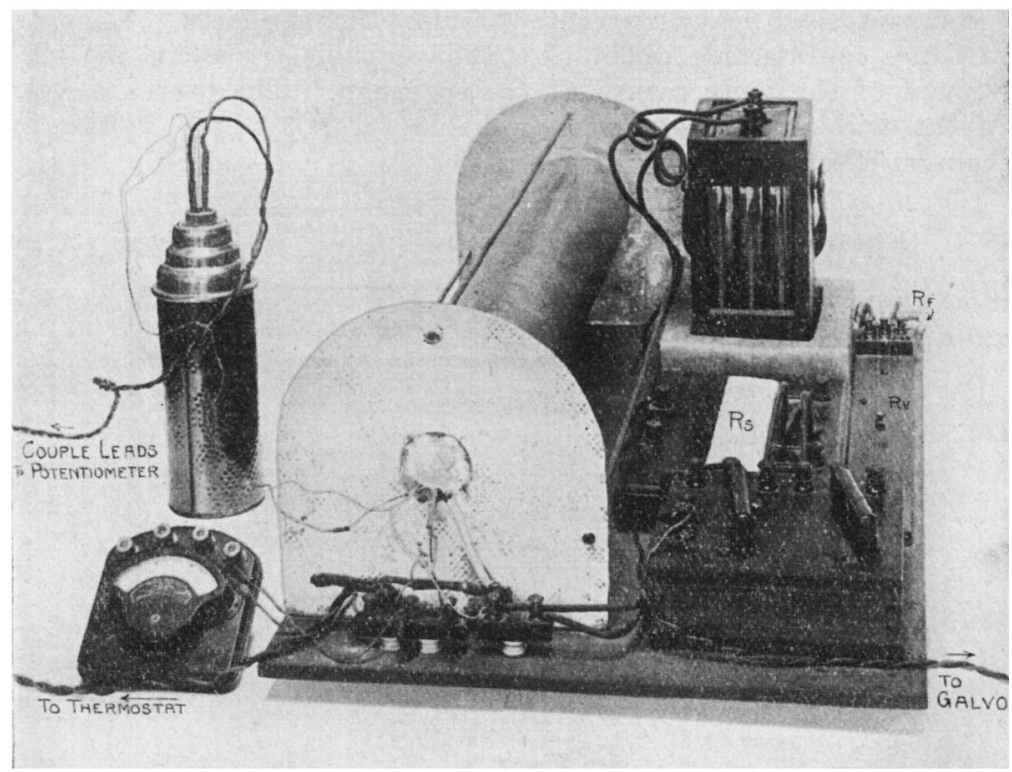

Fig 4

tube. Thespecimen whose temperature-conductivity curve is required, is placed, together with the various leads, thermo-couple, etc., inside the innermost tube, and the open end of the outer clay tube is plugged up with asbestos wool, as is the mouth of the furnace. With the specimen described below, the temperature of the furnace tube varies by $0.5^{\circ} \mathrm{C}$. over the length of the specimen, but the temperature gradient in the specimen itself will be very much less.

The temperature of the furnace can be controlled by hand by means of an external resistance, but a preferable method is to connect it in parallel with a thermostat whose temperature is caused to fall slowly by the method described by Mr. Hanson and the author in an earlier paper.*

A photograph of the apparatus is shown in Fig. 4.

* Further Notes on a High Temperature Thermostat. Haughton and Hanson. Journal of the Institution of Metals, Vol. I8, No. 2, I9I7, p. 17. 
Various forms of specimens have been used. Where possible the metal was cast into a rod from which a sample, of the shape shown in Fig. 5, was turned. Small iron rods were screwed into the specimen in the positions marked $\mathrm{AA}$ and $\mathrm{BB}$, and to the former the current leads were soldered with silver solder, while the potentia] leads were similarly attached to BB.

Specimens of this shape are not suitable or are unobtainable, when it is desired to extend the temperature-conductivity curve above the solidus, or when the specimen is not machinable. Attempts were made to suck the metal (in the case of low melting point alloys) into a glass tube into the sides of which four platinum wires had been sealed. This was not very satisfactory, as it was found exceedingly. difficult to avoid fracturing the glass either during filling or in the subsequent cooling. The best method used consisted in casting a rod about $2 \mathrm{~mm}$. in diameter, and firmly binding on to this four pieces of fine wire (platinum, copper and constantan have been used, according to the circumstances). When the melting tem. perature of the alloy was to be exceeded, the specimen and the wires were given a wash of "Purimachos" cement and, when this had set, further coats of the cement were put on until a solid tube was

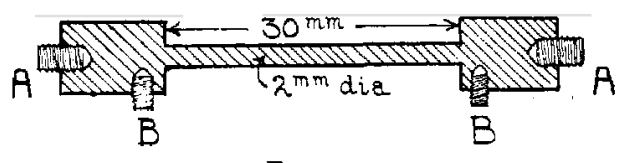

FIG. 5.

built round the specimen. This was then placed inside the innermost tube in the furnace, which was packed with powdered china. clay to reinforce the "Purimachos" tube. This method was found perfectly satisfactory.

The casting of the rods was carried out in two or three different ways, but perhaps the most satisfactory was found to be a method due to Broniewski. This consists in placing some lumps of the metal whose resistance is to be determined in a crucible, into which is inserted the end of a tube of silica or glass, the other end of which is sealed up. The crucible and tube are then placed in a suitably designed electric resistance furnace, and the whole is placed in a vessel which can be evacuated. The air is pumped out of the apparatus, and, as the end of the tube is open in the crucible, this is also evacuated. The furnace is then switched on, and when the alloy melts, the open end of the tube sinks below the surface of the liquid metal. (If necessary, the tube can be weighted to ensurethis sinking.) Air is then admitted into the vessel, and the molten metal is thereby forced up into the tube. The latter is then removed from the furnace, and is broken off the specimen, which remains: in the form of a rod of the same diameter as the bore of the tube.

The majority of the work which has been done with this apparatus is in connection with the investigation of alloy systems, the results: of which are about to be published elsewhere. It is therefore not possible to give many illustrations of the results obtained, but reference may be made to two. 
In an investigation of the constitution of the copper-aluminium. zinc alloys it was found that the liquidus and solidus occur, over a short range, so close together that it was not possible to distinguish between them by means of thermal curves. The liquidus was determined by this means, and the solidus by quenchings, but this meant that the determination of the two lines was carried out on different samples, by different methods, and the various errors introduced might all be additive. Electrical conductivity measure-

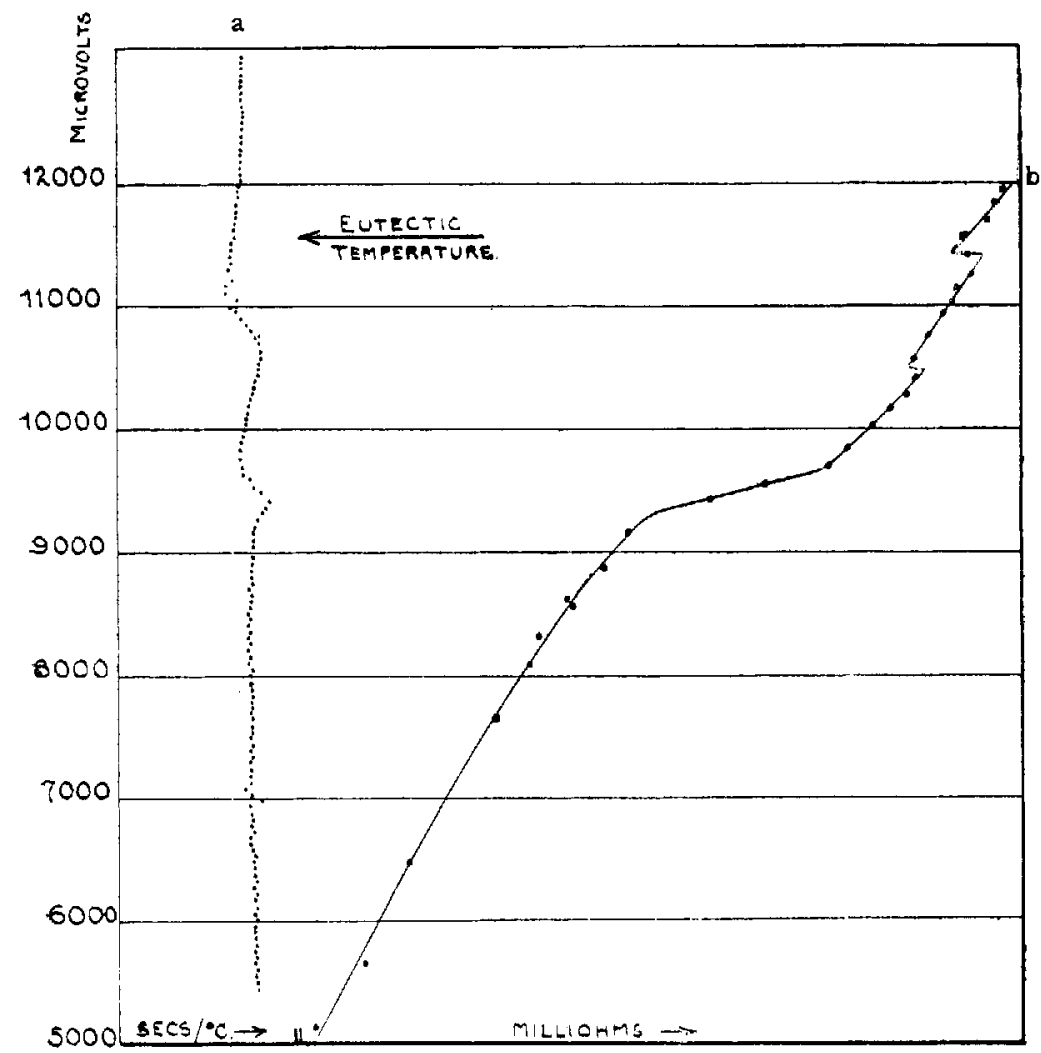

FIG. 6

ments, however, showed marked changes in the direction of the curve connecting conductivity and temperature at both the solidus and the liquidus, and served as a valuable check on the other determinations.

In Fig. 6 a is a thermal curve (heating) of a copper-tin alloy containing about 60 per cent. of tin, and $b$ is an electrical conductivity curve of the same alloy. It will be seen that the latter has three breaks; two of them are also shown in the thermal curve; the lower one of these is probably due to an allotropic modification in one of the constituents of the alloy. The meaning of the other is not clear. The third point on the electrical conductivity curve, 
IN JETALS AND ALLOYS AT HIGH TEMPERATURES 401

which does not show on the thermal curve, is of particular interest. It accurs at the eutectic temperature of the system. Microscopic examination shows that there are the merest traces of eutectic present in the alloy-not nearly enough to show on the thermal curve, but it can readily be seen on the conductivity curve.

In conclusion the author wishes to express his thanks to Dr. Rosenhain, F.R.S., for his interest in the work, and to Mr. Albert Campbell and Mr. S. W. Melson for their advice in connection with the method used for measuring the resistance. 